\title{
The effect of antioxidant supplementation in the treatment of epilepsy
}

\author{
Nazar A.K. hamid \\ Department of Pharmacology, Mosul College of Medicine, University of Mosul
}

$\frac{\text { Received }}{\text { r. } 1 \cdot .2010 \quad r \vee .1 \Upsilon .2010}$

\section{ABSTRACT}

Aim: To assess serum level of malondialdehyde (MDA) and total antioxidant status (TAS) as a representative of oxidative stress in patients with generalized epilepsy and to evaluate the therapeutic effect of the antioxidant (vitamin $\mathrm{E}$ and vitamin $\mathrm{C}$ ) on the levels of MDA,TAS and frequency of seizures attacks after two months therapy. for a period of two months as a supplementation therapy.

Subjects and Methods: The study was conducted in Iben-seena Hospital in Mosul city-Iraq. Fifty three patients with generalized epilepsy were included in this study (32 male and 21 female). The study included 40 apparently healthy subjects, age and sex matched as a control group. Initially from both the patients and controls, blood samples were taken. Another blood samples were taken from the patients 2 months after vitamin $\mathrm{E}$ and vitamin $\mathrm{C}$ treatment, blood samples were analysed for serum MDA and serum TAS.

Result: Serum MDA was found to be significantly higher $(\mathrm{P}<0.001)$ and serum TSA was significantly lower $(\mathrm{P}<0.001)$ in patients with generalized epilepsy prior vitamin $\mathrm{E}$ and vitamin $\mathrm{C}$ supplementation in comparison to controls. After vitamin $\mathrm{E}$ and $\mathrm{C}$ supplementation there was a significant reduction $(\mathrm{P}<0.001)$ in the serum MDA levels with a significant increase $(\mathrm{P}<0.001)$ in the serum TAS. Also we found a reduction in seizure frequency of greater than $70 \%$ after vitamin $\mathrm{E}$ and $\mathrm{C}$ supplementation.

Conclusion: a significant reduction of TSA was reported in patients with epilepsy. Administration of vitamin $\mathrm{E}$ and $\mathrm{C}$ produced a significant reduction of serum MAD levels and a significant elevation of serum TAS, associated with a reduction of greater than $70 \%$ of seizure frequency. The study suggests the administration of vitamin $\mathrm{E}$ and $\mathrm{C}$ as adjunct to antiepileptic drugs.

Key word: Epilepsy, oxidant /antioxidant status, vitamin E, vitamin C.

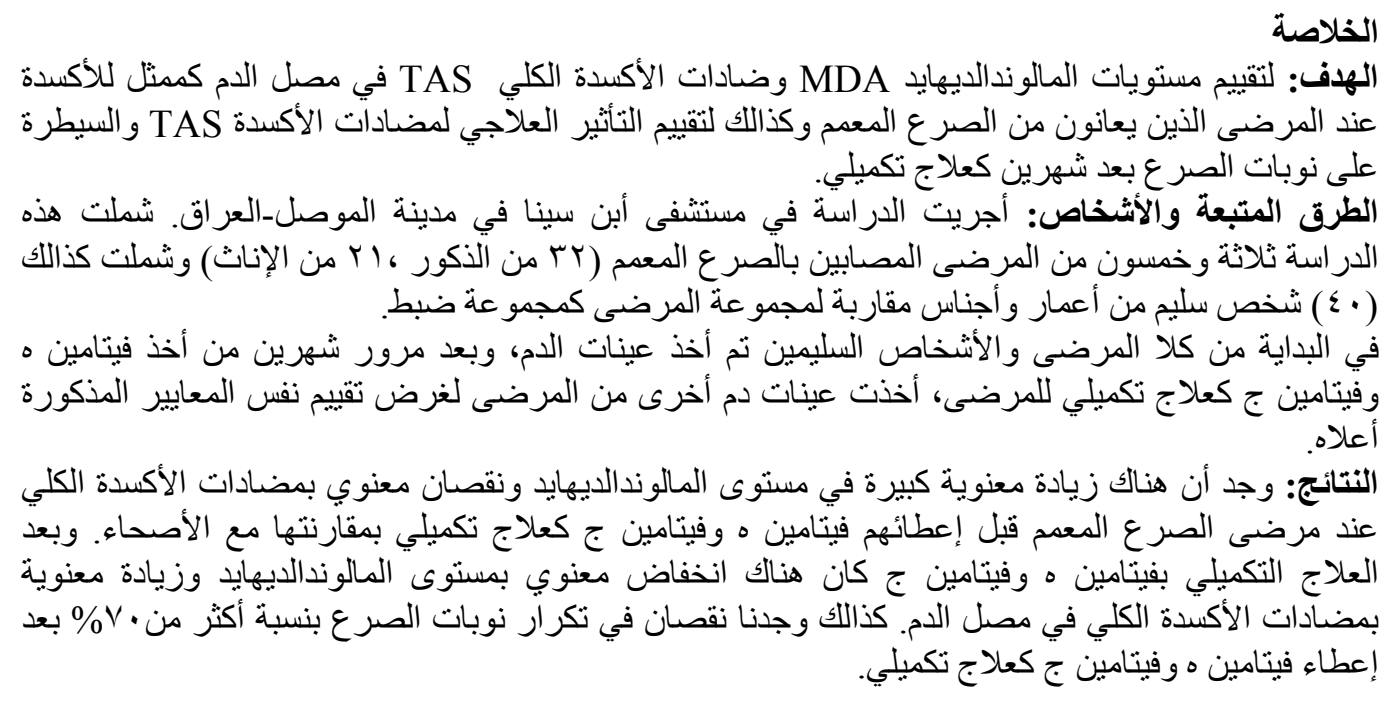




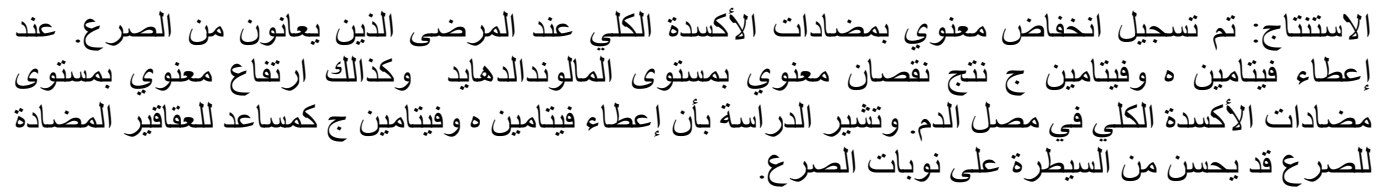

E pilepsy is a chronic disorder seizures $^{1}$. Epilepsy is the second common chronic neurological condition seen by the neurologists ${ }^{2}$. Approximately $60 \%$ of all epilepsies are idiopathic or cryptogenic. Almost any type of brain pathology can cause seizures / epilepsy. The etiology of seizure is multifactorial in any given individual and is best thought of as an interaction between genetically determined seizures thresholds, underlying predisposing pathologies or metabolic derangements and acute precipitating factors ${ }^{3}$.

Oxidative stress is implicated in the pathogenesis of malignancy, dementia, and several neurodegenerative disorders ${ }^{4}$. In mammalian cells, oxygen is reduced to water to generate energy. The reduction of oxygen to water by mitochondrial cytochrome oxidase involve the formation of four single electrons that may combine with oxygen to form reactive oxygen species (ROS) or free radicals. Under pathological condition, approximately $2 \%$ of oxygen does not undergo complete reduction in the mitochondria, which result in the formation of free radicals as the final reaction products ${ }^{5}$. Antioxidant are natural or synthetic substances that may quench the free radicals as soon as they are formed so that the ROS do not get a chance to damage the vital components of DNA or other cellular components. Mammalian cells are equipped with both enzymatic and nonenzymatic antioxidant defiance mechanism to minimize the cellular damage resulting from interaction between the cellular constituents and the ROS. In a healthy body, the ROS and antioxidants remain in balance. When the balance is disrupted toward an overabundance of ROS, a state of oxidative stress prevails when lipid with multiple carbon-carbon double bonds may undergo peroxidation ${ }^{6}$. It is one of the pro-oxidant pathways and a source of free radicals.

The brain is particularly vulnerable to oxidative damage due to the high utilization of inspired oxygen, the large amount of easily oxidizable polyunsaturated fatty acid, the abundance of redox-active transition metal ions, and the relative dearth of antioxidant defense systems ${ }^{7}$.

It has been postulated that membrane lipid peroxidation may be casually associated with certain types of epilepsy. A decrease in free radical scavenging activity may lead to an increase risk of seizure recurrence ${ }^{8}$.

Lipid peroxidation is an indicator of free radical metabolism and oxidative stress in human beings and other organisms. Malondialdehyde (MDA), an end product of lipid peroxidation, is a metabolite that can be readily estimated in serum samples. Our objective, in this study, was to ascertain the variation in serum MDA and serum TAS in epileptic patients before and after antioxidant supplementation.

Vitamin C (ascorbic acid) is one of the antioxidant needed by all cells in the body. Ascorbic acid is a reducing agent and can reduce, and thereby neutralize, reactive oxygen species such as hydrogen peroxide. Anticonvulsant therapy seems to have a negative influence on plasma level of vitamin $\mathrm{C}^{10}$.

Vitamin E ( alpha tocopherol ) is a powerful antioxidant that prevent the 
perioxidation of lipid in the cell membrane. Epileptics on anticonvulsant medication may have reduced plasma alpha tocopherol levels and this may be due to in part to the use of anticonvulsants ${ }^{11}$.

The sensitivity of brain tissue to oxidative damage and the effect of free radical in epilepsy led us to consider the relation between oxidative stress and epilepsy before and after antioxidant supplementation.

The aim of the present study is to evaluate the therapeutic effect of antioxidant supplementation in the treatment of epilepsy

\section{Material and methods}

This study was conducted in IbenSeena Hospital-Department of neurology. All patients included in the study were clinically diagnosed as generalized epilepsy by specialist in neurology. We excluded patients with other disease known to affect oxidant/antioxidant status as diabetes mellitus, hypertension, renal disease, liver disease or malignancy. Also we excluded patients in whom vitamin $\mathrm{E}$ and vitamin $\mathrm{C}$ are contraindicated.

Out of 70 patients 53 patients were selected and completed the study. They were 32 male and 21 female with a mean \pm SD age of $25.24 \pm 10.40$ years (ranged between 14 and 52 years). Also 40 apparently healthy subjects, age and sex matched with patients participated as a control group. They were 23 male and 17 female with a mean \pm SD age $26.93 \pm 10.71$ years.
Consequently, all patients presented at least twice during the study period. They had been treated with anticonvulsants either by monotherapy or combination therapy (Table 1). The drugs used were carbamazepine, valproic acid, Phenobarbital, phenytoin, clonazepam and topiramate.

All patients were given 400 I.Us/d vitamin $\mathrm{E}$ and $100 \mathrm{mg} / \mathrm{d}$ vitamin $\mathrm{C}$ for 2 months. Initially ( before vitamin $\mathrm{E}$ and $\mathrm{C}$ administration ) blood samples were taken from the patients and controls for the assessment of serum MDA and TAS. After 2 months of antioxidant supplementation, another blood samples were taken from patients for reassessment of MDA and TAS.

Assay of MDA was done manually using a method described by Onkawa et al, ${ }^{12}$, TAS was measured by peroxidase/ $\mathrm{H}_{2} \mathrm{O}_{2}$ /ABTS colorimetric assay using commercial kits from Pandox company- UK.

The data were expressed in mean \pm SD. Statistical analysis was done by using student 's ' $t$ '-test and $\mathrm{p}<0.05$ was considered as significant and $\mathrm{p}<0.001$ was considered as highly significant.

\section{Results}

A significant higher level of MDA, and lower level of TSA were obtained in patients before vitamin $\mathrm{E}$ and $\mathrm{C}$ administration as compared to control group $\quad(\mathrm{p}<0.001) \quad($ Table-2)

Table 1. Overall Antiepileptic drugs (AEDs) utilization (N=53)

\begin{tabular}{|l|l|l|l|}
\hline S.No & AED therapy & No of patients & percentage \\
\hline 1. & Monotherapy & 27 & $51 \%$ \\
\hline 2. & Dual therapy & 21 & $40 \%$ \\
\hline 3. & Three drugs & 5 & $9 \%$ \\
\hline
\end{tabular}


Table 2. Concentration of MDA and TSA in epileptic patients before vitamin E and C supplementation and healthy control (mean \pm SD)

\begin{tabular}{|l|l|l|l|}
\hline Parameter & $\begin{array}{l}\text { Patients before vitamins } \\
\text { supplementation } \\
\text { No=53 mean } \pm \text { SD }\end{array}$ & $\begin{array}{l}\text { Control } \\
\mathrm{No}=40 \\
\text { mean } \pm \text { SD }\end{array}$ & P value \\
\hline $\mathrm{MDA}(\mathrm{nmol} / \mathrm{l})$ & $1.91 \pm 0.21$ & $0.72 \pm 0.16$ & $\mathrm{P}<0.001$ \\
\hline $\mathrm{TSA}(\mathrm{mmol} / \mathrm{l})$ & $1.13 \pm 0.14$ & $1.70 \pm 0.18$ & $\mathrm{P}<0.001$ \\
\hline
\end{tabular}

Table 3. Concentration of MDA and TAS in patients with epilepsy after vitamin E and $\mathrm{C}$ supplementation and healthy control (mean $\pm \mathrm{SD})$

\begin{tabular}{|l|l|l|l|}
\hline Parameter & $\begin{array}{l}\text { Patients before vitamins } \\
\text { Supplementation } \\
\text { No=53 mean } \pm \text { SD }\end{array}$ & $\begin{array}{l}\text { Patients after vitamins } \\
\text { Supplementation } \\
\text { No=53 mean } \pm \text { SD }\end{array}$ & P value \\
\hline MDA $(\mathrm{nmol} / \mathrm{l})$ & $1.91 \pm 0.21$ & $1.79 \pm 0.20$ & $\mathrm{P}<0.001$ \\
\hline TAS $(\mathrm{mmol} / \mathrm{l})$ & $1.13 \pm 0.14$ & $1.30 \pm 0.14$ & $\mathrm{P}<0.001$ \\
\hline
\end{tabular}

Table 4. Frequency of improvement of epilepsy after vitamin E and C supplementation

\begin{tabular}{|l|l|l|l|l|}
\hline Degree of control & $\begin{array}{l}\text { No of } \\
\text { patients }\end{array}$ & $\begin{array}{c}\text { Percentage } \\
\%\end{array}$ & $\begin{array}{c}\text { MDA } \\
\text { mean } \pm \text { SD }\end{array}$ & $\begin{array}{c}\text { TAS } \\
\text { mean } \pm \text { SD }\end{array}$ \\
\hline Good control & 19 & $36 \%$ & $1.67 \pm 0.17$ & $1.38 \pm 1.10$ \\
\hline Fair control & 29 & $55 \%$ & $1.79 \pm 0.22$ & $1.26 \pm 0.15$ \\
\hline Bad control & 5 & $9 \%$ & $1.88 \pm 0.16$ & $1.26 \pm 0.08$ \\
\hline
\end{tabular}

After 2 months of vitamin $\mathrm{E}$ and $\mathrm{C}$ therapy, a significant reduction of MDA and a significant elevation of TSA were obtained (Table 3).

Data obtained from the study showed that a reduction in the frequency of seizure attacks have been produced after one month of vitamin $\mathrm{E}$ and $\mathrm{C}$ supplementation. 19 patients (36\%) presented total remission of seizure. $29 \%$ patients $(55 \%)$ presented improvement with reduction of seizure by $60 \%-90 \%$ and 5 patients $9 \%$ did not respond to treatment (Table 4).

\section{Discussion}

Brain is considered abnormally sensitive to oxidative damage and in fact early studies demonstrated the ease of peroxidation of brain membranes. Brain is enriched in the more easily oxidizable polyunsaturated fatty acid such as decosahexaenoic acid and eicosapentaenoic acid as it has a limited ability to perform aerobic glycolysis, it is unusually vulnerable to hypoxia. On other hand, brain is not enriched in antioxidant defenses, it contain relatively low levels of superoxide dismutase, catalase and glutathione peroxidase ${ }^{13}$.

Imbalance in oxidants and antioxidants known to be involved in the pathogenesis of various diseases including epilepsy. Although the specific underlying cause of epilepsy and seizures is often unknown, research has found that damage caused by free radicals can predispose the brain to seizures. The high fat content of myelin sheaths that surround neurons and the high rate of oxidative 
metabolism (about 20\% of the total oxygen demand of the body) make the brain a target for free radical damage. Many factors can induce excessive production of free radicals, including head trauma and neurodegenerative disease $^{14}$.

Studies have shown that epileptics are low in many antioxidants, including intrinsic antioxidants such as glutathione and superoxide dismutase and extrinsic antioxidants including vitamin $\mathrm{E}$, vitamin $\mathrm{C}$, and vitamin $\mathrm{A}^{15}$. Although large human studies have not yet been conducted on the use of antioxidants in people with epilepsy, it is already known that vitamin $\mathrm{A}, \mathrm{C}$ and $\mathrm{E}$ are vital to brain function ${ }^{16,}$.

In the present study, one of the indices of oxidative stress, serum MDA, and TAS, were assessed in 53 patients with generalized epilepsy and 40 healthy controls before and after antioxidant supplement. Our study demonstrated a statistically significant increase in the levels of MDA with statistically significant reduction in TAS in patients with epilepsy before vitamin $\mathrm{E}$ and vitamin $\mathrm{C}$ supplementation as compared to control group. After vitamin E and vitamin $\mathrm{C}$ supplementation we observed significant reduction in serum MDA levels with an increase in serum TAS from pre-supplementation values.

This study indicated that serum TAS of epileptic patients, which was low compared with controls, improved after treatment, suggesting that free radicals may be implicated in epilepsy.

These finding are in accordance with several studies which has been done to evaluate oxidative stress and antioxidant status in various types of epilepsy. Most of these studies have shown enhanced level of oxidative stress and reduced levels of antioxidant vitamins $^{17,18}$.
In this study, we examined the effect of vitamin $\mathrm{E}$ and vitamin $\mathrm{C}$ supplementation on 53 patients. At the end of 2 months we found a dramatic decrease in seizure activity in these patients. In small trial using vitamin $\mathrm{E}$ in 24 children aged 5-18 years with refractory epilepsy, treatment of 12 children with vitamin E, $400 \mathrm{IU} / \mathrm{d}$, over 3 months improve seizure control in 10 children, indicated by a reduction in seizure frequency of greater than $60 \%$ compared with a 3 months run-in baseline period ${ }^{11}$. Some other studies also showed that vitamin $E$ supplementation reduced seizure frequency, although no improvement was seen in other studies ${ }^{19}$.

Vitamin $\mathrm{C}$ is an exogenous antioxidant able to alter the brain oxidative stress. In one study found that vitamin $\mathrm{c}$ increase the latency to first seizure and decrease the mortality rate and lipid peroxidation levels in adult rats with seizures induced by pilocarpine $^{19}$.

Adding vitamin $\mathrm{C}$ to certain drugs help them penetrate the brain and may improve treatment of neurological disease. Many medicines that could potentially help in diseases such as Alzheimer's disease, Parkinson's disease and epilepsy are limited by their inability to reach the brain. That is because the brain is protected from foreign' substance by a natural barrierthe blood -brain barrier. They attached vitamin $\mathrm{C}$ to a drug that could be used to treat epilepsy but cannot, alone, reach the brain. Mice with epilepsy showed no improvement when treated with the drug but when vitamin $\mathrm{C}$ was attached, there was a significant reduction in seizures. the researchers think this works because vitamin $\mathrm{c}$ does pent rate the brain through vitamin c 'transporters' found in brain tissue. When a drug is linked to vitamin, they can use the transporters 
as a hook to pull them through the blood-brain barrier ${ }^{20 .}$

In conclusion, the ability of antioxidants for reducing the seizures manifestations and the accompanying biochemical changes (i.e makers of oxidative stress) further supports a role of free radicals in seizure and highlight a possible role of antioxidants as adjunct to antiepileptic drugs for seizure control.

\section{References}

1. Leppik I E. Contemporary diagnosis and management of the patient with epilepsy, $2^{\text {nd }}$ ed. Newtown, PA: Hand books in health care. 1996.

2. Sridharan R. Epidemiology of epilepsy. Cur Scie 2002;82(6):664-70.

3. Guberman AH, Bruni J editors. Essentials of clinical epilepsy. $2^{\text {nd }}$ ed. Boston, Butterworth Heinemann,. 1999. P. 3-10.

4. Halliwell B, Gutteridge JM, editors. Free radicals in Biology and Medicine. Oxford: Oxford University press 1997.

5. Fridovich I. Superoxide dismutase. Adv Enzymol 1974;41:35-97.

6. Rice-Evans C, Burdon R. Free radical-lipid interactions and their pathological consequences. Prog Lipid Res 1993;32:71-110.

7. Butterfield DA, Castegna A, Pocernich CB, et al. Nutritional approaches to combat oxidative stress in Alzheimer's disease. J Nutr Biochem 2002;13:444-61.

8. Maertens P, Dyken P, Grf W, et al. Free radicals, anticonvulsants and the neuronal ceroid lipofuscinosis. Am J Med Genet 1995; 57:225-8.

9. Shigeoka S, Ishikawa T, Tamoi $\mathrm{M}$, et al. Regulation and function of ascorbate peroxidase.
Isoenzymes. $\mathrm{J} \quad \operatorname{Exp} \quad$ Bot 2002;53(372):305-19.

10. Singh RB, Niaz MA, Ghosh S, et al. Dietary intake and plasma levels of antioxidant vitamin in health and disease: A hospital based case controlled study. J Nutr Environ Med 1995;5:23542.

11. Ogunmekan AO, Hwang PA. A randomized, double-blind, placebo-controlled, clinical trial of D- $\alpha$-tocopherol acetate (vitamin E), as added-on therapy, for epilepsy in children. Epilepsia 1989;30:84-9.

12. Ohkawa H, Ohishi N, Yagi K. Assay for lipid peroxides in animal tissue by thiobarbituric acid reaction. Anal Biochem 1979;95:351-8.

13. Guney Y, Bilgihan A, Hiesonmez A, et al. Influence of different doses of irradiation on oxidant and antioxidant systems in the brain of guinea pigs. Am J Immunol 2005;3:114-8.

14. Halliwell B. Role of free radicals in the neurodegenerative disease: Therapeutic implications for antioxidant treatment. Drugs Aging 2001;18(19):685-716.

15. Liao KH, Mei QY. Determination of antioxidants in plasma and erythrocyte in patients with epilepsy. Zhong Nan Da Xue Xue Bao Yi Xue Ban 2004;29(1):72-4.

16. Almeida SS, Duntas LH, Dye L, et al. Nutrition and brain function: A multidisciplinary virtual symposium. Nutr Neurosci 2002;5 (5): 311-20.

17. Sudha K, Rao AV, Rao A. oxidative stress and antioxidant in epilepsy. Clin Chim Acta 2001;303(1-2):19-24.

18. Golden TR, Patel M. Catalytic Antioxidants and 
Neurodegeneration. Antioxid Red Sign 2009;11(3):555-70.

19. Gaby AR, National Approach to Epilepsy. Alternative Medicine Review 2007 vol. 12 No 1: 9-24.
20. Aldridge S. Vitamin C could improve brain drugs. J Med chemi; Jan 31. 2002/ online edition, December 21,2001. 